\title{
Inhibiting roles of FOXA2 in hepatocellular carcinoma cell migration and invasion by transcriptionally suppressing microRNA-103a-3p and activating the GREM2/LATS2 axis
}

\section{Guangzhen $\mathrm{Ma}$}

the Second People's Hospital of Liaocheng

Jirong Chen

Suizhou Hospital Affiliated to Hubei Medical College

Tiantian Wei

Suizhou Hospital Affiliated to Hubei Medical College

Jia Wang

Suizhou Hospital Affiliated to Hubei Medical College

Wenshan Chen ( $\nabla$ wenshan010501@163.com)

Suizhou Hospital Affiliated to Hubei Medical College https://orcid.org/0000-0003-1221-325X

\section{Research}

Keywords: FOXA2, miR-103a-3p, GREM2, LATS2, YAP, Hepatocellular carcinoma

Posted Date: August 19th, 2020

DOI: https://doi.org/10.21203/rs.3.rs-26285/v2

License: (c) (i) This work is licensed under a Creative Commons Attribution 4.0 International License.

Read Full License 
The authors have withdrawn this preprint from Research Square 